\title{
Emotional Regulation
}

\section{Report on the Study of Social and Emotional Skills of Chinese Adolescents (II)}

\author{
Zhi Liu, Ruirui Zhu, Haili Cui, \& Jingzhong Huang \\ East China Normal University, Shanghai 200241, China
}

\section{$\mathrm{R}$}

ECENTLY, an article published in the Journal of East China Normal University (Education Sciences) was based on the data of 10-year-old and 15-year-old students in Suzhou City, China participating in the OECD social and emotional ability assessment. The study used descriptive statistics, difference testing, regression analysis, and other statistical methods to conduct a comprehensive analysis of the emotional regulation performance of Suzhou students.

Emotion regulation is an important part of one's social and emotional ability. In the OECD's research on social and emotional ability, based on the theory of the "Big Five Personalities", the connotation of emotional regulation is defined as whether a person can manage emotional well, effectively adjust negative emotions and cope with pressure, as well as maintain a positive and optimistic attitude towards one's personal life and social career development. In view of the emotional adjustment methods of adolescents and the characteristics that they cannot accurately observe and express, the emotional adjustment dimensions of the OECD Social and Emotional Ability Assessment measure stress resistance, optimism, and emotion control. One carries out the three-demonstration analysis based on the performance of the item's ability. The emotional state will have an important impact on one's study, work, and personal life. A large number of empirical studies indicate that emotional regulation is closely related to health and well-being, but especially overall physical and mental health. The OECD pointed out in its re-

(C) 2021 Insights Publisher. All rights reserved.

(c) (i) (5) Creative Commons Non Commercial CC BY-NC: This article is distributed under the terms of the Creative Commons Attribution-NonCommercial 4.0 License

(http://www.creativecommons.org/licenses/by-nc/4.0/) which permits non-commercial use, reproduction and distribution of the work without further permission provided the original work is attributed by the Insights Publisher. 
search report that emotion regulation is of great significance to the life development of children and adolescents and is closely related to many life outcome variables (such as educational expectations, test anxiety, life satisfaction, happiness, health, etc.). With the increasing uncertainty of current social development, students must face many sudden changes in learning, living environment, and interpersonal relationships. In this case, regulating emotions is particularly important for students. When cannot adapt and adjust, anxiety, depression, pessimism, and other emotions may result. Therefore, it is extremely important and urgent to teach young students to regulate and manage emotions well.

The sampling of the OECD Youth Social and Emotional Ability Research is systematically conducted from all eligible schools and students in the participating cities, specifically to all primary and secondary schools in the 6 districts and 4 county-level cities under the jurisdiction of Suzhou City. The study uses a stratified two-stage cluster sampling method. The 10-yearold group and the 15-year-old group constituted 3,800 and 3,750 subjects, respectively, with the total sample size being 7,550. The research results are divided into three parts, including overall condition, analysis on predictive variables of emotion regulation, and variables of emotion regulation and life outcome. The analysis shows differences in the students' abilities to resist stress, their optimism, and emotional control according to age, gender, urban and rural areas, and school categories. There is a correlation among the 15 abilities of the "Big Five Personalities" model, which one can analyze from multiple levels through regression analysis. One may also explore the predictive variables of students' emotion regulation and the influence of emotion regulation on the five major life outcome variables. The details are as follows:

\section{The Overall Situation of Emotional Regulation}

The overall performance of Suzhou students in emotional regulation reveals the scores of various sub-abilities of emotional regulation, the correlation between emotional regulation and other sub-abilities, and the differences in age, gender, urban and rural areas, and school types of emotional regulation. The study found that among 10-year-old students, anti-stress, optimism, and emotional control were significantly related to other abilities $(\mathrm{p}<0.01)$. Optimism and emotional control were at a medium-to-high level, with an impact coefficient of 0.60. Anti-stress and optimism were at a medium-to-high level. The level-related influence coefficient was 0.57, and emotional control and anti-stress were also related to the middle and high levels, with an influence coefficient of 0.57 . Anti-stress, optimism, and emotional control were significantly related to other abilities $(\mathrm{p}<0.01)$ among students in the 15 -year-old group. Optimism and emotional control were related to a medium-to-high level, with an impact coefficient of 0.62. Anti-stress and optimism were related to a medium-to-high level. The influence coefficient was 0.60. Emotional control and anti-stress were also related to the middle to high level, and the influence coefficient was 0.57 . 
There were also significant differences in the score performance of various sub-abilities of emotion regulation in age, gender, urban and rural areas; the differences between general high school and vocational high school were insignificant. There is a substantial difference between the age-based tripartite evaluations of students, parents, and teachers. The evaluations of the 10year-old group by students and teachers are 0.08-66 points higher than those of the 15-year-old group, while the parents' evaluations of the 10-year-old group are lower than $1511-23$ points in the age group. With the increase of age, the difference in the ability of male and female students in the dimension of emotion regulation will change. In the lower age group, there is little difference between boys and girls, but in the higher age group, boys have better emotional regulation ability than girls. Students in the central city indicate better emotional regulation than that of the rural students. The urbanrural differences in the three abilities of anti-stress, optimism, and emotion control in the 10-year-old group are statistically significantly different from 0 ( $\mathrm{p}<0.01$ ), indicating the central city students' emotional regulation is significantly higher than in rural areas. The 15 -year-old students have relatively small differences in the mean of these 3 abilities, and there is no statistically significant difference.

\section{Analysis of Predictors of Emotion Regulation}

It is necessary to use multiple regression to analyze the factors that affect emotional regulation development, including background variables, student variables, teacher variables, school variables, and family variables.

Regarding the background variables, in the 10-year-old group of students, the two variables of family possessions and the number of family books have stable and significant positive effects on the three emotional regulation abilities. The $\mathrm{P}$ values are all less than 0.01 , and the coefficients respectively are 0.24 . 0.09. In the 10-year-old group of students, the three variables of parents' highest education, family possessions, and family book collection have stable and significant positive effects on the three abilities of emotional regulation. The $\mathrm{P}$ values are all less than 0.01 , and the coefficients are $0.01,0.17,0.01$, respectively.

Regarding student variables, in general, whether it is a 10-year-old or a 15-year-old student, the six variables of security, friendship, good habits of friends, friendliness of classmates, outdoor activity time and growth-oriented thinking are all related to emotional regulation. The item ability has a significant and stable positive impact $(\mathrm{p}<0.01)$, and a wide range of social relationships negatively impacts the 10 -year-old group of students $(\mathrm{p}<0.01)$ and positively impacts the 15 -year-old group of students $(\mathrm{p}<0.01)$.

Regarding teacher variables, for the 10-year-old group of students, the two variables of teacher-student relationship and teaching age have stable and significant positive effects on the three abilities of emotional regulation dimensions ( $\mathrm{p}<0.01$ ), with coefficients around 0.18 and 0.05 . For the 15year-old students, the three abilities of the teacher-student relationship in the 
emotional regulation dimension have stable and significant positive effects ( $\mathrm{p}$ $<0.01$ ), with a coefficient of about 0.16 . Whether it is a 10 -year-old or a 15 year-old student, the teacher-student relationship can have a stable and significant positive impact on the three abilities of stress, optimism and emotional control. In addition, for the 10-year-old group of students, the teacher's high expectations have a negative impact on students' emotional regulation $(\mathrm{p}<0.01)$, but for the 15-year-old group, similar effects are not obvious $(\mathrm{p}=$ $0.01)$.

Regarding school variables, for the 10-year-old group of students, the three variables of school belonging, school cooperation atmosphere, and extra-school activities have stable and significant positive effects on the three abilities of emotion regulation ( $\mathrm{p}<0.01$ ), with $0.32,0.15$, and 0.09 coefficients, respectively. For 15-year-old students, similar to 10-year-old students, the three variables of school belonging, school cooperation atmosphere, and extra-school activities have stable and significant positive effects on the three abilities of 15-year-old students in the emotional regulation dimension ( $\mathrm{p}<$ 0.01 ), with coefficients of $0.40,0.05$, and 0.09 , respectively.

Regarding family variables for the 10-year-old group of students, the three variables of understanding fathers, understanding mothers, and parents' high expectations have stable and significant positive effects on the three emotional regulation abilities $(\mathrm{p}<0.01)$ with coefficients of $0.14,0.11$, and 0.06 , respectively. Parent-child problems and punitive fathers have a significant negative impact on the three abilities $(\mathrm{p}<0.01)$, and the coefficients are 0.17 and 0.00 , respectively. This shows that the discordant parent-child relationship and strict fathers hinder students' emotional regulation abilities. For the 15-year-old group of students, the three variables of understanding fathers, punitive fathers, and parents' high expectations have stable and significant positive effects on the three abilities of emotional regulation dimensions ( $p<0.01$ ), with coefficients of $0.11,0.05$, and 0.07 , respectively. The difference in the 10-year-old group is that the strictness of the father's discipline positively correlated to a child's ability to regulate emotions. The degree of influence of understanding parents is generally higher than that of punitive parents, and for 15-year-old students, the influence of fathers is greater than that of mothers. Moreover, punitive mothers have a negative influence on children's emotional regulation. In addition, consistent with the 10-year-old group, the parent-child problem also has a significant negative impact on the three abilities $(\mathrm{p}<0.01)$, with a coefficient of about 0.23 .

\section{Emotion Regulation and Life Outcomes}

From the perspective of academic performance and educational expectations, for the 10-year-old group of students, the optimism of emotional regulation and emotional control has a stable and positive impact on students' performance in Chinese, Mathematics, and Art $\mathrm{p}<0.01$ ). In contrast, the influence of optimism is greater (coefficients are 0.06, 0.06, 0.05, respectively). Compared with students in the 15 -year-old group, optimism and emotional con- 
trol have different effects on the performance of each subject and their educational expectations. From the perspective of global awareness, whether it is a 10-year-old, or a 15-year-old student, anti-stress, optimism, and emotional control have a positive and significant impact on their global awareness ( $\mathrm{p}<$ 0.01). This indicates that improving anti-stress, optimism, and emotional control could increase the global awareness of students of different age groups. From the perspective of health, whether it is a 10-year-old or a 15year-old student, the emotional regulation ability has a significant positive impact on one's health status ( $\mathrm{p}<0.01$ ), indicating that one's optimism, ability to resist stress and emotional control can enhance one's health. From the perspective of quality of life, whether it is a 10-year-old or a 15-year-old student, the ability to resist stress, optimism and emotional control has a significant positive impact on happiness and satisfaction $(\mathrm{p}<0.01)$, and a significant negative for test anxiety influence $(\mathrm{p}<0.01)$, that is, the better the emotional regulation ability, the less test anxiety. From the perspective of social relations, the assessment of social and emotional abilities mainly includes variables such as being close to family members (parents) and close to others (companion relations). From the analysis results, in both groups, emotional regulation ability has a significant positive impact on close family members and others to whom one is close $(\mathrm{p}<0.01)$, indicating that the ability to resist stress, optimism, and emotional control can be improved. These abilities can make students closer to family and others.

Therefore, as an important dimension of social and emotional ability, emotion regulation is affected by different subjects and many variable factors. Under the current rapidly developing and changing social background, it becomes increasingly important for students to have strong emotional regulation abilities. Accordingly, this study presents some research recommendations: In terms of concepts, we must attach great importance to cultivating students' emotional regulation-related abilities; in implementation, we must master and use strategies and models for emotional regulation; all relevant subjects must clarify their own roles, explore effective paths, and form a joint force for educating people. We should also promote the improvement of students' emotional regulation levels.

Source: Journal of East China Normal University (Educational Sciences), 2021, 39(09): 47-46. 